Session

\title{
Assessing applications for Solid Modeling (CSG) in Architectural education
}

\author{
Dr. Stan G. Guidera \\ College of Technology \\ Bowling Green State University, Bowling Green, Ohio
}

\begin{abstract}
This paper discusses the functionality of solid modeling and proposes approaches to utilizing solid modeling to develop course content that emphasizes the conceptual applications of computer modeling over application-specific CAD coursework. More importantly, the paper discusses approaches for structuring these activities to emphasize the integration of content covered in other courses. Assignments from an introductory 3D modeling course are used to illustrate how solid modeling was used to foster content integration from architectural history and construction methods courses.
\end{abstract}

\section{Introduction}

Competency in computing has become a pre-requisite for securing employment in architectural practice. However, Architecture schools have been criticized for failing to provide sufficient technical education and preparation for the realities of practice [1]. While deficiencies in knowledge-development related to construction and building technologies has often been a specific point of criticism [2], schools have more recently been challenged with developing a strategy to address skills related to digital media in general and CAD skills more specifically. While it has been proposed that the mandate of architectural education is to cultivate skills in life-long learning and that practice is where students develop technical knowledge [3], this is at least to some extent inconsistent with the reality that "technical competence in computing technology has become a condito sine qua non of landing a job at a respectable architectural practice"[4]. The challenge faced by architectural educators is highlighted by the ever increasing scope of content that must be addressed, including CAD skills, and, according to Mark [5], " a central problem to educational programs is how to make room for computer-related technology and at the same time not replace time-honored parts of the curriculum."

Much of the focus on computing in architectural curriculums has been on the role of $\mathrm{CAD}$ and digital media in support of design studio. CAD can provide an effective and sometimes necessary tool to facilitate creation of complex forms that may not be buildable or even documented using conventional representation tools [6] [7], and the embrace of computer modeling in the design studio among some students and educators is a logical extension of the widely publicized utilization of computing tools by "star" architects and designers. 
CAD is typically taught independently from studio and that the established studio tradition prevalent in architectural education contrasts with the typical skill-building approach to teaching CAD in which computer skills are taught in a way that is parallel with other classes such as drawing, drafting, or model-making [5]. Mark argued that "the skill-focused means of teaching is detrimental to the development of appropriate skills and attitudes in CAAD application" and that "this mode of teaching reinforces the perception that CAAD is a technique and tool that is separate from architecture." In contrast to skill-focused teaching, a position statement by eCAADia 2001 proposed that there should be an integration of CAAD education into architectural curriculum and that CAD education should be taught during the whole span of architectural education [5]. Similarly, a white paper by the Association for Computer Aided Design in Architecture (ACADIA) stated that "students need to use digital techniques throughout the curriculum" and that "a digital design sensibility must pervade the school culture" [8].

Given the predominance use of discrete courses to deliver content related to CAD in many architecture curriculums, an alternative approach to integrating digital skills may be to utilize the discrete CAD courses as a mechanism for integration. Rather than emphasizing the role of CAD as a tool for design studio, this approach would emphasize the use of CAD as a tool to reinforce knowledge and content from other coursework. Such an approach may be more effectively served by de-emphasizing application specific CAD skills and addressing skills that may be transferable between applications in order to focus on the related content. For classes that are structured to develop three-dimensional computer modeling skills, content based on the use of Solid Modeling using Constructive Solid Geometry, or CSG, provides a unique opportunity to promote interoperability of skills between applications.

\section{Solid modeling and architecture}

A "Solid Model" is defined as "an unambiguous computer representation of a physical solid object" [9]. Computer modeling with solids that is based on Constructive Solid Geometry (CSG) is a method for describing the geometry by applying set operations to primitive objects [10]. CSG is used to build solid models by combining primitive (implicit) objects with Boolean operators. Solid model primitive objects, such as spheres, cubes, and cylinders are manipulated with Operators to produce new objects based on addition, intersection, and union of the primitive objects.

The Boolean Set Operators used are:

- Union $-\mathrm{A}+\mathrm{B}$ is the set of points that are in $\mathrm{A}$ or $\mathrm{B}$.

- Intersection - A.B is the set of points that belong to A and B.

- $\quad$ Difference - A-B is the set of points that belong to A but not to B [11]. (Figure 1.)

In addition to Constructive Solid Geometry, 3D CAD applications include the ability to create complex solid-based geometry by sweeping two-dimensional shapes and projections [12]. These extrusions and swept solids, such as a revolution or linear sweep of a planar face, can also be used in Boolean operations. Constructive solid geometry (CSG) uses trees (CSG Trees) to track the operations on the building block primitives. Conceptually, objects that compose the CSG tree are represented with the root of the tree defining the object, the terminal branches or 
nodes of the tree corresponding to the geometric primitives, and the non-terminal nodes corresponding to the Boolean Set operations that are used to create the final model. (Figure 2.)

The utilization of conventional solid modeling has been limited in architecture. This can be attributed to factors that include technical limitations. For example, due in part to the number of modeled components typically associated with architectural projects, the use of solid modeling can generate prohibitively large file sizes as the geometric complexity of a model increases. Additionally, the lack of robustness associated with rounding-off and approximation errors due to the extensive use of approximation in geometric constructions in solid modeling remains an obstacle with many software applications. This also has implications for the ways in which designers interact with their models in that modeling robustness requires that models are "always semantically interpretable and retain design intent during their entire life cycle"[13].

The limitations of solid modeling to support an iterative process that can flexibly access and re-interpret design intent from earlier design stages is one of the reasons that solid modeling has been perceived as a technology that is disconnected with the conceptualization processes associated with architectural design [14]. As a result, professional architectural CAD applications have evolved from the early versions of CAD modeling, based primarily on surfaces and extrusions, to the current releases of software based on object-oriented modeling technologies. Although in many of these applications solid modeling provides the underlying technology for many of the modeling commands, there is only limited use of conventional primitive-based solid operations.

While this has been a positive advance in software development for architectural practice, this position overlooks characteristics of solid modeling that can be more appropriately exploited in academic settings, particularly with introductory 3D modeling courses. Three of these characteristics can be of particular interest to educators. First, creating computer models with constructive solid geometry is not inherently application-specific. Methodology and terminology, such as the use of Boolean operations for union-subtraction-intersection modeling, are common to a wide range of modeling applications. For example, AutoDesk's AutoCAD includes a Box, Cone, Sphere, Cylinder, Wedge, and Torus as solid primitives Objects, and uses Union, Intersection, and Subtraction as nomenclature for primary Boolean operations. Form Z by Auto.Des.Sys also provides a Box, Cone, Sphere, Cylinder, and Torus as solid primitives Objects. Form $\mathrm{Z}$ also uses the terms Union and Intersection to describe two of the three primary Boolean operations, but uses the term Difference in lieu of Subtraction. Similarly, Micro Station Modeler refers to Boolean operations in terms of Union, Subtraction (Difference), and intersection and includes tools for creating primitives such as rectangular solids (slabs), Spheres, Cones, Torus shapes (donuts), Wedges, and Cylinders. This commonality facilitates transferability of knowledge and skills between software products and platforms.

Secondly, rather than application-specific commands required to utilize the features of more advanced object-oriented CAD software, solid modeling processes utilize a more limited palate of commands. This can reduce the learning curve by allowing students to concentrate on a narrower range of menus and command syntax and also promotes transferability of knowledge. AutoCAD uses the term slice to refer to the process and command used to divide or cut Boolean objects. Form $\mathrm{Z}$ utilizes the term Split rather than Slice, the command operations associated with 
these commands parallels those utilized for in AutoCAD. As with common use of terminology, this commonality facilitates transferability of knowledge and skills between software products and platforms.

Lastly, the role of primitive volumes in CSG modeling is aligned with fundamental architectural design concepts. There is a historical precedent for the use of pure geometric volume as a theoretical and perceptual underpinning in architecture. Eighteenth century design proposals by Boulee and Ledoux utilized pure geometric volumes as primary formal design elements (Figure 3). Illustrations of these theoretical projects, which were based on the use of platonic solids, are commonly found in architectural history and theory texts [15][16][17]. Other historical references to geometric forms in architecture that parallel CSG primitives can be found in statements by LeCorbusier, who praised the aesthetic qualities of pure geometric form:

"Cubes, cones, spheres, cylinders, or pyramids are the great primary forms that light reveals to advantage; the image of these is distinct and tangible within us and without ambiguity. It is for this reason that these are beautiful forms, the most beautiful forms" [18].

Ching also discussed the role of platonic solids, the sphere, cylinder, cone, pyramid, and cube, in terms of perceptual and architectural qualities. His discussion also referenced the manipulation of 2D shape as a basis for generating platonic solids:

"The primary (two-dimensional) shapes can be extended to generate volumes whose terms are distinct, regular, and easily recognizable. These forms are referred to as the platonic solids. Circles generate spheres, triangles generate cones and pyramids, squares generate cubes" [19].

More importantly, the emphasis of solid modeling on form and volume can facilitate the exploration of architectural and spatial concepts that are often more commonly associated with more traditional design and studio courses. Spatial modeling, also referred to as modeling "negative space" refers to the concept of modeling a volume or void as an object or form, in contrast with the more conventional approach of modeling enclosing surfaces such as walls, floors, and ceilings. The concept of negative space has a traditional association with architecture and is often introduced in fundamental design courses as a way to assist students in conceptualizing space and volumes. Arnheim [20] described negative space as "the empty spaces within and around the material volumes" and added, "the function of negative space is strengthened by the fact that in architecture the open spaces are the territory of the human occupant." Negative space modeling with CAD has been utilized in manufacturing and mechanical design as a tool for modeling the voids in which parts are to fit [21] and Mitchell and McCullough suggested that solid modeling could be utilized as a spatial representation tool in architecture [15].

Based on these characteristics of solid modeling, a discrete CAD course was revised to include tasks specifically designed to integrate knowledge from the content areas of other architecture courses within the curriculum of a four-year undergraduate degree in architecture. The following case study discusses the organization, objectives, and outcomes of the solid- 
modeling course assignments in terms of CAD skills as well as effectiveness in reinforcing content knowledge from related courses.

\section{Structuring an Integrative CAD class}

The course in this case study was the second of two required CAD courses. All students in the class were architecture majors and were at varied levels of academic experience. An AutoCAD-based engineering graphics course with content emphasizing two-dimensional CAD skills and competency with basic drawing, editing, and data-organization was a pre-requisite for the class. As a result, all students enrolling in the course were assumed to have fundamental CAD skills but no experience or formal training in computer modeling.

The course was organized to utilize the first three course projects to develop competencies with three dimensional computer modeling. These initial three projects were also structured to integrate content and drew on content from construction materials and methods, architectural history, and architectural design concepts. Although AutoDesk Architectural Desktop, a parametric object-oriented programming CAD application, was used in the course, no parametric features of the software were used in these assignments. All of the integrative activities utilized CSG-based solid modeling with class demonstration and lab activities structured to develop competencies in modeling with the following:

- Boolean operations (Union, Subtraction, Intersection)

- Directional and rotational extrusions of 2D shapes

- Path extrusions of 2D shapes

- Navigation and manipulation of 3D work planes

- Moving, modifying, and placing CAD objects and forms in virtual space

- Manipulation of the 3D display of virtual objects to communicate a concept.

The activities were hierarchical in terms of the modeling skills required to complete the assigned tasks, with each project involving higher levels of competencies with solid modeling and manipulating 3D geometry. Each project was structured with activities organized in two phases and included class-time allocated to reviewing and discussing topics and information related to the content that was to be integrated with the CAD exercises. In addition to the projects, bi-weekly quizzes were used to assist in assessing the students. Examples of the activities and associated objectives for each of these content areas are discussed in the following sections.

\section{Integrating Construction Materials and Methods}

The construction materials and methods project was the initial integrative assignment in the course. The primary skill set required included directional extrusions, and required students to create three-dimensional models that documented the construction of wall assemblies. Each student was required to select a unique construction assembly and all assemblies were required to be modeled using only solid modeling. Project parameters stated that the CAD models were to be developed at full-scale and that the dimensions of all materials represented in the model were to reflect the actual dimensions of materials. 
The first phase for Project One required students to research material form and sizes and develop conventional orthographic representations of the wall assembly. This not only reinforced 2D CAD skills but also enabled the instructor to work with the students to ensure the assembly documentation accurately reflected the correct dimensions and assembly. The second phase required the students to use solid modeling techniques to create the three-dimensional model. The modeling activities utilized moving and placing CAD objects and forms in virtual space and directional extrusions of 2D shapes. The assignment required limited use of Boolean operations and path extrusions. (Figure 4)

Building the assembly also required the manipulation of the 3D display of virtual objects, primarily through the use of predefined isometric and orthographic views. However, a secondary activity, a sequential display task, was used to emphasize conceptual communication and make additional connections to the construction materials and methods content. This task required the students to assign each construction component to a discrete layer in order to control that component's display independently. Students were then required to research the sequence of activities associated with the construction of the assembly, and produce a presentation that reflected the sequence of construction. (Figure 5.)

\section{Integrating CAD with Architectural History}

The Architectural History assignment was the second integrative assignment in the course and was used to develop competencies with more advanced CAD concepts. Building on the skills introduced in the initial class activities, this activity involved extensive use of Boolean operations and directional and rotational extrusions of 2D shapes as well as the skills associated with path extrusions of 2D shapes, navigation and manipulation of 3D work planes, and moving and placing CAD objects and forms in virtual space. As in the construction materials and methods assignment, the first phase of Project Two, which overlapped the modeling phase of Project One, required students to conduct out-of-class research. Each student was assigned a separate building of historical architectural significance from the classical Greek, Roman, and Renaissance periods. The structures were pre-selected by the instructor based on the availability of sufficient documentation as well as the compatibility of the structure's form with the modeling techniques the assignment was intended to utilize. Additionally, the models developed in Project Two were also used in the activities in Project Three. Therefore, the pre-selection was also based on the compatibility of the structures spatial and volumetric characteristics.

After completing the initial research, each student prepared a written report and developed 2D CAD representations of the plan, sections, and elevations of the structure. The 2D representations were used to document dimensional information related to their assigned structure and for developing an understanding of the structure's architectural form and character. While this knowledge was used as a starting point for the development of the mass model, the research, documentation, and report component also served to reinforce the connection to architectural history courses.

The emphasis of the computer-modeling component of Project Two was primarily focused on the use of additive Boolean operations. Tasks were organized sequentially; with the initial tasks requiring students to produce a massing that approximated the three-dimensional 
form of their assigned structure. However, detailing the mass model required students to also utilize subtraction and intersection Boolean operations as well as directional, rotational, and path extrusions of 2D shapes. (Figure 6).

Associated classroom activities for Project Two also included several instructordeveloped tutorials that were designed to help students develop required CAD skills while further reinforcing the connection between the computer modeling assignment and aspects of architectural history (See Appendix A). For example, a tutorial that was intended to introduce students to solid modeling using rotational extrusions led students through a step-by-step process of modeling Doric and Ionic columns, and included within the tutorial a discussion of the classical orders and their prominence in Greek and Roman architecture, and the reinterpretation of the orders in neo-classical architecture. Another tutorial, intended to develop student skills in using more complex solid modeling commands and strengthen skills with manipulation of workplanes, was organized around modeling the dome of Florence Cathedral.

\section{Architectural Design Concepts: Interior Modeling and Negative Space}

The third solid modeling assignment was an extension of Project Two. The first activity for Project Three required students to use Boolean operations to "carve" the interior spaces out of the exterior massing models of their assigned structure (Figure 7). After the dominant spaces were subtracted, more detailed interior elements, such as columns and cornices, were developed and added to the model. A second activity was used to reinforce the student's ability to conceptualization three-dimensional space. Students were required to create a discrete model of the primary volume of their assigned structure. In addition to the skills that were developed in Projects One and Two, activities for Project Three emphasized the use of subtractive and intersection Boolean operations.

\section{Observations and assessment}

Observations of the student success in developing specific CAD skills as well as the extent to which content knowledge from other subject areas was enhanced were documented throughout all three of the project activities. The complexity of each of the three projects was hierarchical, and the parameters of Project One, Integrating Construction Materials and Methods, required students to develop competencies with a relatively limited number of modeling commands and related skills. However, it became apparent that, for several students, there was limited retention of knowledge and skills from the pre-requisite CAD course, which required more class time to be allocated to review than initially planned. Additionally, the classroom experience of the students enrolled in the course varied extensively. Several students had not completed any construction classes and were limited in their knowledge of construction terminology and materials. As a result, it also became necessary to allocate course time to introducing content related to construction rather than to use the project to reinforce knowledge from construction-related courses and experience. This content was covered during the first phase for Project One as the students researched construction materials, selected assemblies to model, and produced conventional orthographic representations of their assemblies. 
All students appeared to master the required modeling tasks and geometric manipulations associated with the project. However, some students had more limited success in producing dimensionally accurate assembles. Similarly, while all students were able to use their models to produce a presentation that documented a construction sequence, it was observed that students with the most accurate models also produced the more detailed construction sequence presentations. Unlike Projects Two and Three, quizzes that were administered during Project One included items that were intended to assess learning related specifically to Construction Materials and Methods. Comparisons between responses to a quiz given prior to the start of the project with one administered later in the course indicated that that nearly all students had increased their knowledge related to construction materials and practices.

The outcomes with Project Two, integrating architectural history, were somewhat more uniform in that there was greater consistency among the students in meeting the project parameters. Review of the written report, intended to help students to understand the historical context of their assigned structure, indicated that it also assisted students in identifying important features and characteristics that were essential to be incorporated in their models. There was a direct association between the level of detail produced in the 2D documentation phase and the level of detail of the final three-dimensional models produced.

While Project Two required students to utilize a wider range of commands, it also was less proscribed in terms of the modeling strategies that could be utilized. In Project One, students produced most of their wall assembly models in a systematic order, beginning with foundations and moving up the structure in an order that to a large extent paralleled real-world construction processes. It was observed that although students appeared to be able to utilize the more complicated commands associated with Project Two, some students had varying degrees of difficulty in developing an effective strategy for starting their models. A common approach was to start with plan shapes and building up with extrusions rather than approaching the structure as a combination of form associated with the solid primitives. The students who were most successful in producing detailed and proportionately accurate models utilized the primitives to create a general massing of their assigned structure, and then used more complex solid operations to articulate the massing.

The outcomes observed for Project Three paralleled those of Project Two. At this point in their skill development, students had developed sufficient competencies with the command set to complete the project activities. As in Project Two, there was a direct association between the students who were most successful in producing accurate negative space models and the level of detail produced in the 2D documentation phase. More specifically, it was observed that students who developed multiple sections rather than the single section required in the $2 \mathrm{D}$ documentation assignment were able to more effectively model the interior spaces. As in Project Two, some students approached the modeling of the interior volumes by extruding plan shapes rather than as a mass assembled with solid primitives. The most proportionate and accurate interior models and negative space models utilized solid primitives as the primary modeling strategy and then refined their models with path extrusions and revolved profiles.

Complications with software operations were reported during the activities associated with both Project Two and Project Three. As the level of detail of models increased, students 
reported problems with computer performance, including decreases in performance associated with display and navigation, longer time for file-saves, and system crashes during operations. These complications were likely a result of the large file sizes associated with solid models. More specifically, because solid models retain the history of the operations used to create the model, the use of repetitive Boolean operations required to develop a higher level of detail inherently results in very large file sizes. Additionally, rendering an image of a model from a CSG tree can be slower than other model geometries because CSG representations must be converted to a B-rep before rendering the display [11]. Therefore, it is likely that geometric complexity of the more detailed solid models contributed to the navigation and display problems experienced by several students, particularly as they attempted to work on their models in shaded display modes.

\section{Conclusions and recommendations}

An integrative CAD course requires direct connections to other content areas in the curriculum. Although the outcomes of the course indicated that the use of solid modeling in a CAD course to reinforce knowledge from the other content areas could be successful, it was also evident that the curriculum design can itself be a limiting factor in meeting this goal. Organizing the curriculum to insure that students have the required or expected knowledge base is necessary to maximize the effectiveness of the CAD class in reinforcing the related content.

It is also recommended that greater consideration be given to hardware and software limitations in structuring assignments. For example, it is likely that decreasing the emphasis on the level of detail required in Project Two and Project Three could reduce the likelihood of the performance experienced by students without compromising the assignment objectives. Additionally, it is also recommended that the assignments incorporate tasks that require students to utilize efficient CAD practices, such as purging unnecessary information from the CAD files in order to decrease demands on system performance.

Lastly, where possible an assessment of the success of the students in meeting the objectives of reinforcing content as well as the transferability of the CAD knowledge should be integrated in the course. The pre and post assessment for Project One, which indicated that students not only met the objectives related to CAD skills but also demonstrated an increased comprehension and retention of the content that was to be reinforced, was an effective mechanism for determining the effectiveness of the integrative activities. The scope of the pre and post assignment assessments should be expanded to include content related to all three projects. While the architectural history-related research activities were an important part of the assessment for Project Two and Project Three, the lack of any pre-assignment assessment made it difficult to more objectively determine their effectiveness in reinforcing knowledge in those content areas.

The extent to which students were able to transfer CAD knowledge skills to other applications should also be assessed. Where available, the utilization of multiple CAD applications should be employed to assess the transferability of knowledge and skills. In the assignments associated with this case study, the inter-related activities of Project Two and 
Project Three would provide a unique opportunity to utilize different software applications for each project in order to provide a basis for assessing transferability.

While the outcomes of the solid modeling assignments indicated they were effective in integrating content from other areas of the architecture curriculum, the suggested modifications could further enhance the development of CAD skills in the context of the full curriculum. More importantly, these outcomes indicate that successful integration of related content with discrete CAD courses suggests that consideration should be given to integrating CAD into other courses such as Construction and Materials and Methods courses. Therefore, if the ACADIA recommendation that "a digital design sensibility must pervade the school culture" in order for educators to realize the potential of CAD and computing, faculty in all content areas should consider developing greater competencies with CAD and related applications. Although this approach places additional demands on faculty, it can serve to foster a more holistic approach to the use of digital media in architectural education.

\section{References}

1. Mitgang, L. "Back to School: Architects Sound Off on 10 Critical Issues Facing Architectural Education." Architectural Record. September, 1999.

2. Mitgang, L. "Saving the Soul of Architectural Education." Architectural Record. May, 1997.

3. Karloff, R. How the Profession is Failing the Schools. Architecture 85 (1996).

4. Tomassian, R., and Marx, J. Digital practices. In Bermudez, J. and Klinger, K. (eds.) Digital technology and architecture-White paper. (2003). www.acadia.org/ACADIA.whitepaper.pdf

5. Mark, E., Martens, B., and Oxman, R. "Preliminary stages of CAAD education." Automation in Construction, 12, pp. $661-670.2003$.

6. Sequin, C. (2001). "CAD and the Arts". Computer-aided design. (33) p.345.

7. Kolarevic, B. (2003). "Digital production/fabrication.” In Bermudez, J. and Klinger, K. (eds.) Digital technology and architecture-White paper. (2003) www.acadia.org/ACADIA.whitepaper.pdf

8. Cheng, N. (2003). "Digital curriculums: Effective integration of digital courses." In Bermudez, J. and Klinger, K. (eds.) Digital technology and architecture-White paper. www.acadia.org/ACADIA.whitepaper.pdf

9. Requicha, A.A.G., Rossignac, J.R. "Solid Modeling And Beyond." CGA(12), No. 5, 1992, pp. 31-44.

10. http://www.hyperdictionary.com/computing/constructive+solid+geometry

11. http://escience.anu.edu.au/lecture/cg/surfaceModeling/CSG.en.html

12. Anderl, R., and Mendgen, R. Parametric Design and its impact on solid modeling applications". Association of Computing Machinery. 1995.

13. Patrikalakis, N. (2000). Robustness issues in geometric and solid modeling. Computer-aided design. (32) p.629.

14. Mitchell, W. and McCullough, M. "Digital Design Media". New York; Van Nostrand Reinhold. 1994.

15. Pevsner, N. "An Outline of European Architecture". Pelican Books 1994

16. Kroft, H. "A history of architetcural theory from Vituvius to the present." Princeton Press. 1994

17. Sources of architectural form: A critical history of western design theory." Manchester University press. 1995

18. LeCorbusier. "Towards a new architecture". Dover Publications. 1986

19. Ching, F. "Form, Space, and Order". Wily \& Sons. 1985

20. Arnheim, R. "To the rescue of art: Twenty-six essays". Berkeley: University of California Press. 1992.

21. Welsh, W. "Introduction to CAD-driven reverse engineering". Society of Manufacturing Engineers IMTS Conference. September 7, 2000.

STAN GUIDERA is an Associate Professor in Architectural/Environmental Design Studies in the College of Technology at Bowling Green State University. He teaches design studios, advanced CAD courses and computer rendering and animation. He is a registered architect and has used computer-aided design extensively in professional practice. 


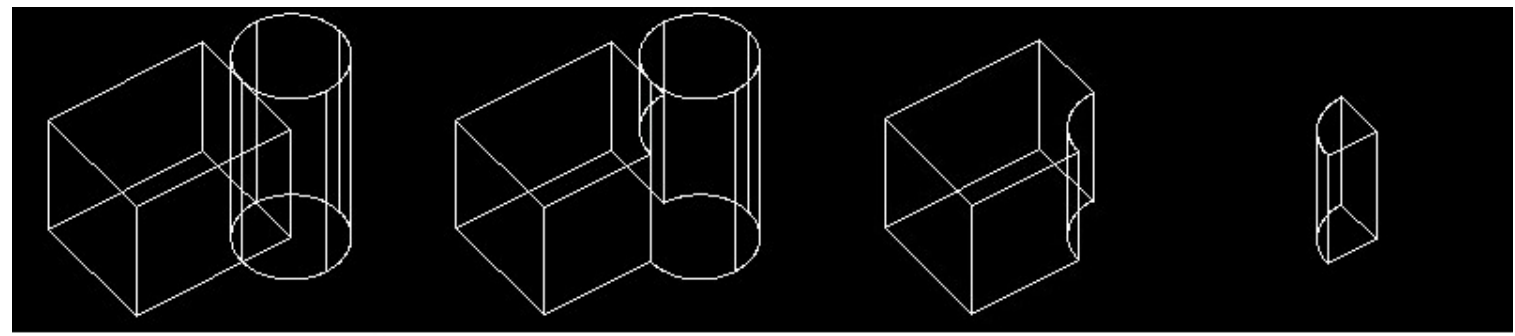

Boolean Operators:

Cube (Operand A)

Union:

Subtraction:

Cylinder (Operand B)

Cube (Operand A) +

Cylinder (0perand $B$ )

Cube (Operand A) -

Intersection:

Cylinder (Operand B)

Common volume

of $A$ and $B$

Figure 1. Boolean Set Operations

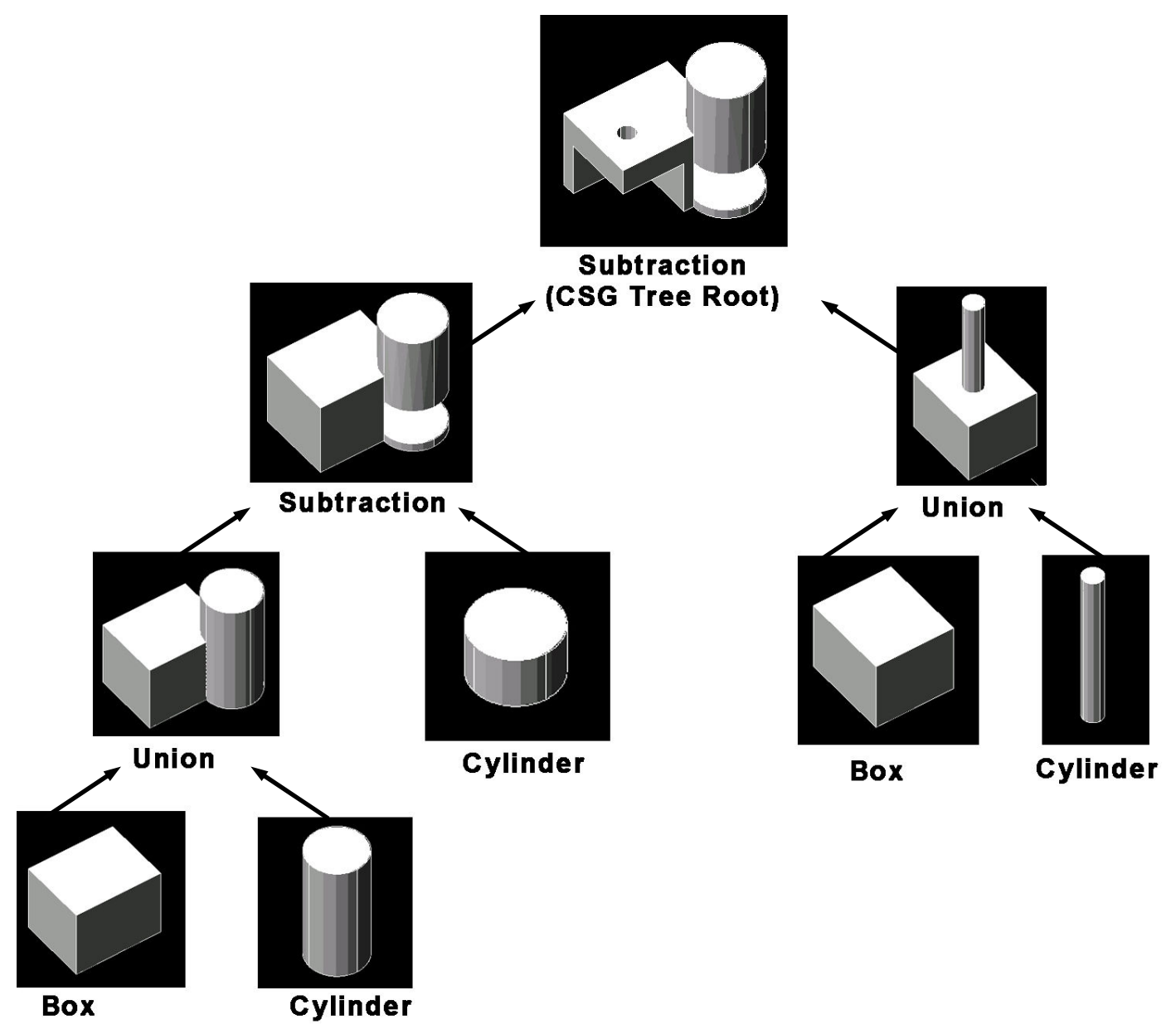

Figure 2: CSG Tree Diagram

"Proceedings of the 2005 American Society for Engineering Education Annual Conference \& Exposition Copyright (c) 2005, American Society for Engineering Education" 

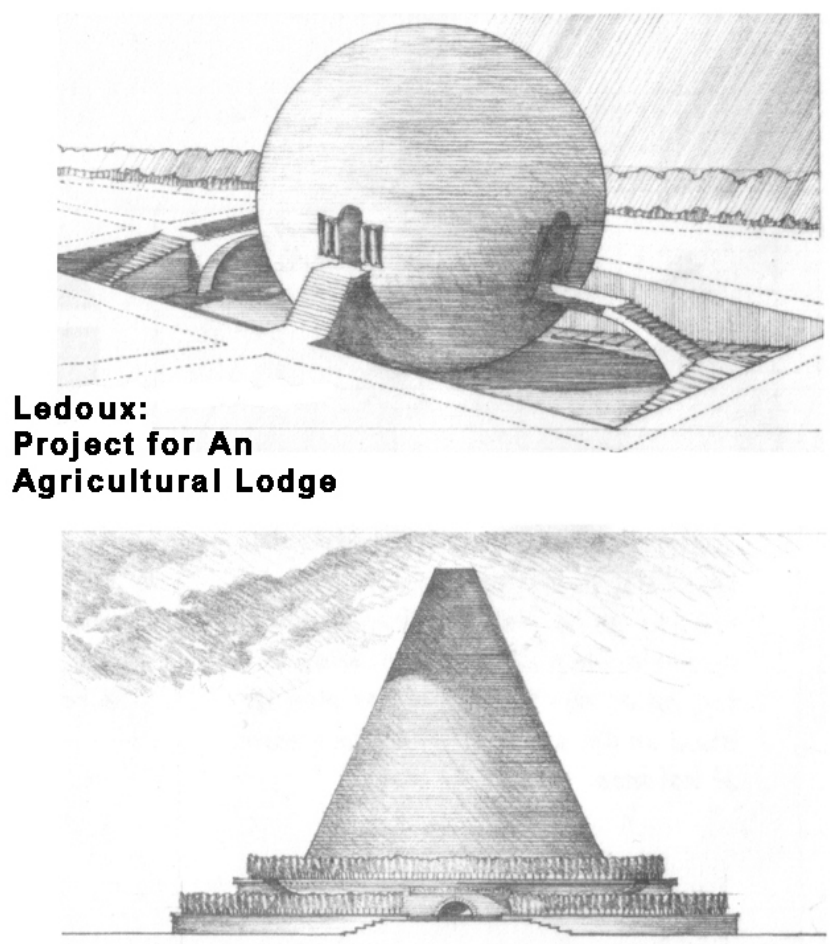

\section{Boulee: \\ Conical Cenotaph}

Figure 3: Projects by Claude Nichololas Ledoux and Etienne Louis Boulee From: Form, Space and Order by Francis Ching

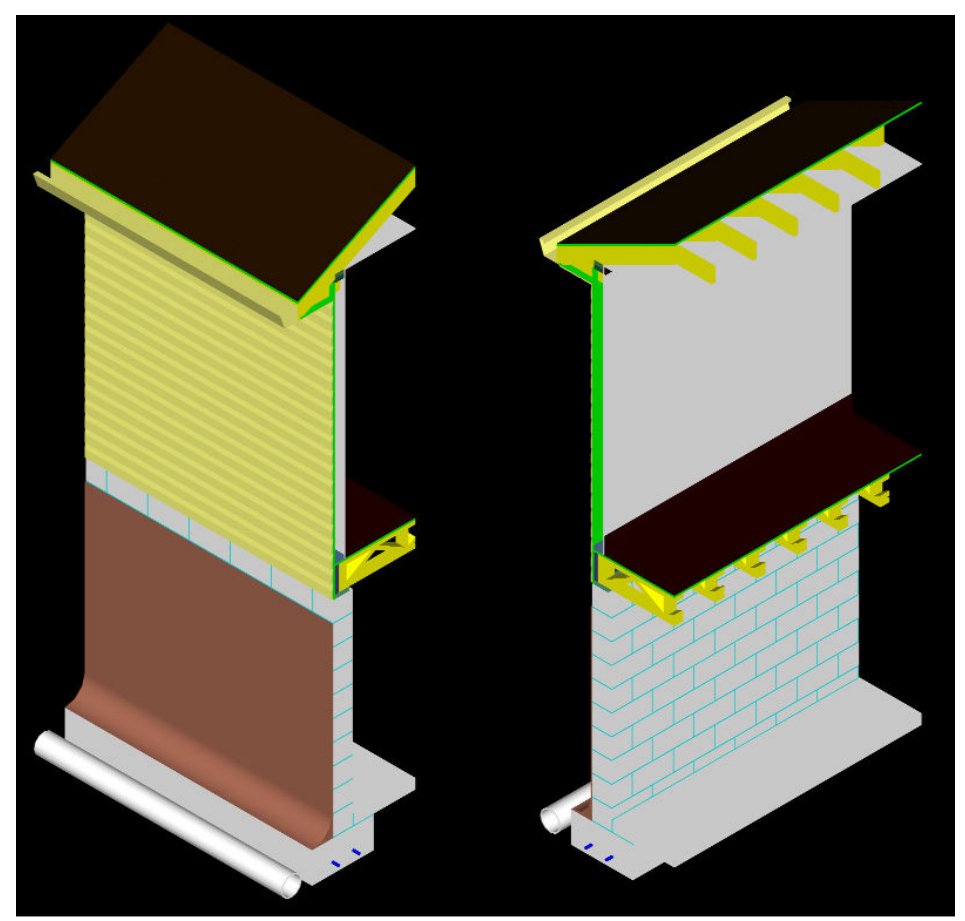

Figure 4: Student Submission, Project One: Wall section solid model composition

"Proceedings of the 2005 American Society for Engineering Education Annual Conference \& Exposition Copyright (c) 2005, American Society for Engineering Education" 


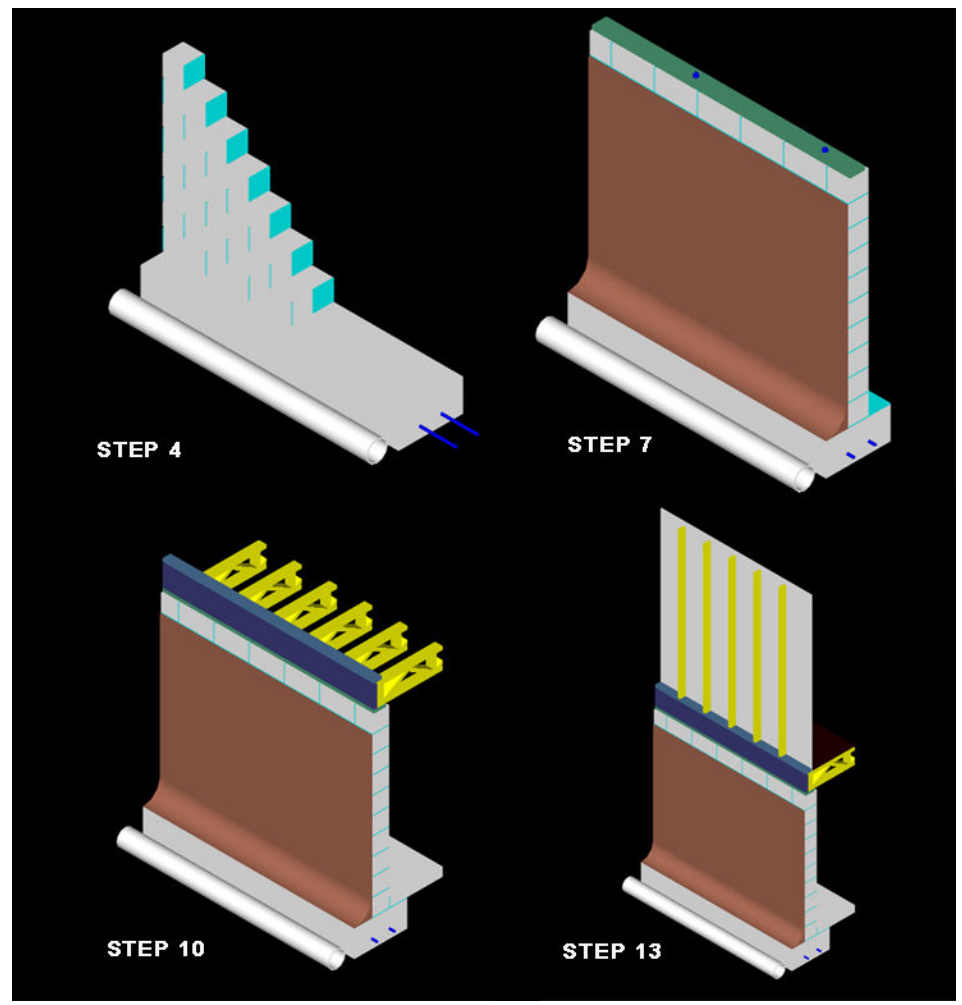

Figure 5: Student Submission, Project One: Construction Sequence Presentation composition

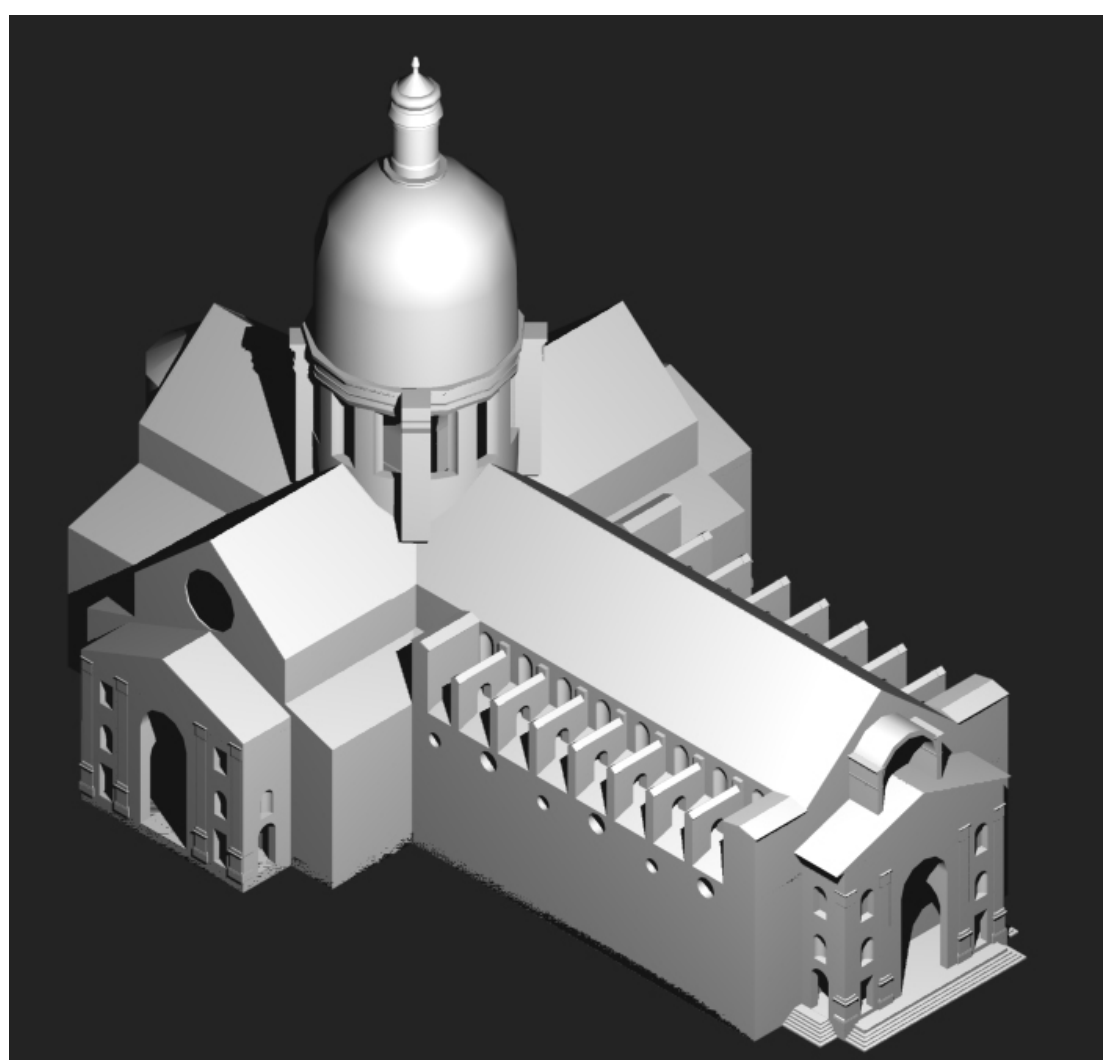

Figure 6. Project Two Student Submission: Detailed mass model

"Proceedings of the 2005 American Society for Engineering Education Annual Conference \& Exposition Copyright (C) 2005, American Society for Engineering Education" 


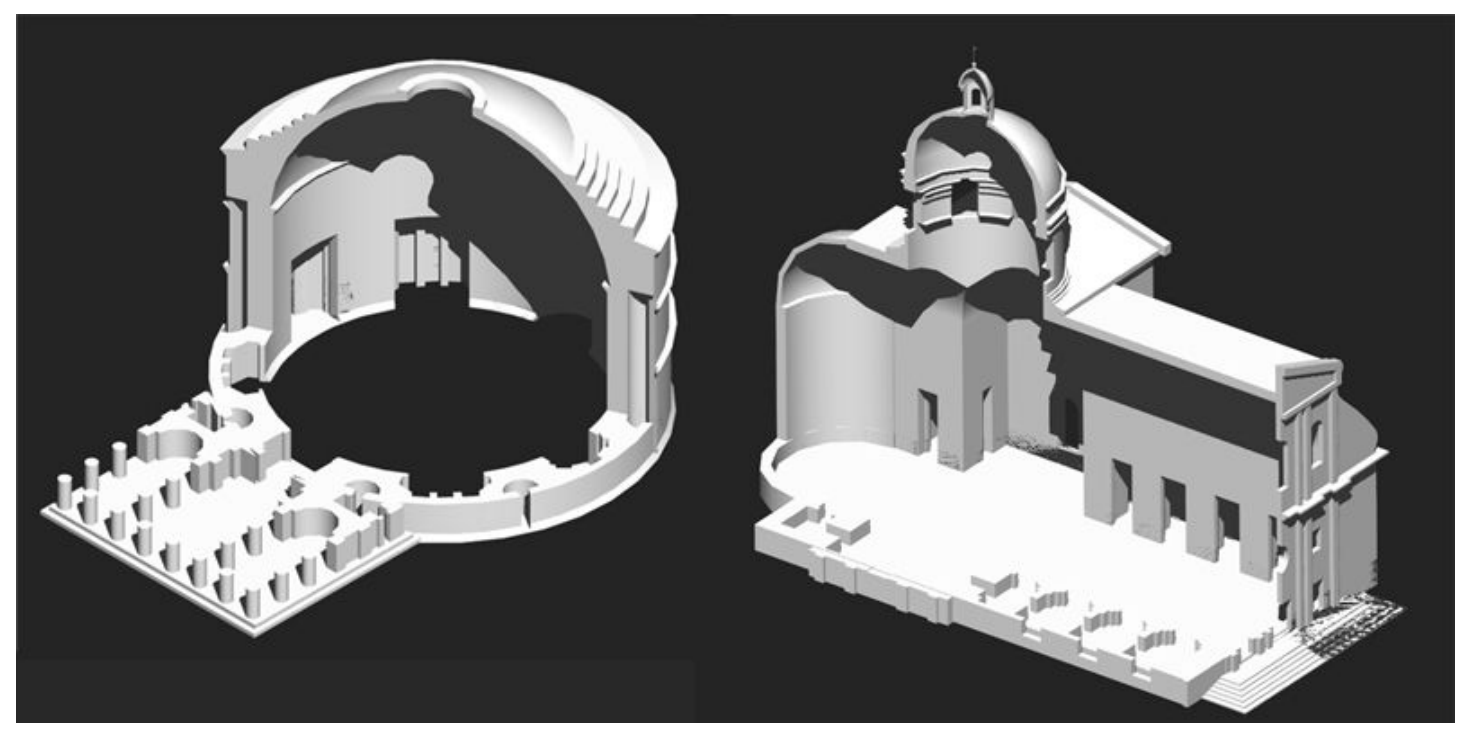

Figure 7. Project Three, Student Submission: Interior model.

Appendix A: Tutorial Introduction

\section{Building the Dome \\ The significance of Florence Cathedral, also known as San Maria del Fiore, in renaissance architecture is attributed in part to the construction of its dome. Construction on Florence Cathedral began in 1296. The original design by Arnolfo di Cambio was modified by Francesco Talenti . By 1366 Talenti's design called for an enormous octagonal dome. However, the construction of the dome posed significant challenges to the known building techniques of the 14th century. According to Raeburn, "The diameter of the dome at 39.5 metres (130 feet) precluded the traditional use of wooden structuring to support the construction of the vault, while the use of buttresses as in northern Gothic cathedrals was ruled out by the building's design." In 1420 Fillipo \\ Brunelleschi's proposed technical solution for construction of the dome vaulting was approved and construction of the dome began. The church was consecrated in 1436.}

Architecture 271 Tutorial 3: Modeling the Dome of the Florence Cathedral

The dome of Florence Cathedral is actually a very innovative element. It is in effect an arched and tapered octagon. However, its geometry does not lend itself to using a revolved shape or an extrusion along a path (if you are so inclined I encourage you to try to create it using these methods - it would be a very good learning experience. Although some of the dimensions are available, the scale on the drawings was used to estimate dimensions. As always there are other alternatives to the approach we will be taking here but the end result should produce a fairly reasonable representation of this architectural element. At the end of this handout is a sketch as well as the dimensional and pictorial information for Florence Cathedral, taken from Sir Banister Fletcher's A History of Architecture on the Comparative Method (New York: Scribners Press). Using the scale on the section, the dimensional information was estimated and used to produce the sketch of the section we will use as the basis for the following exercises. After completing Tutorial 3, you should understand how to creating 3D solid extrusions using paths, be able to apply the slice command using points on existing geometry, and perform Boolean operations to create complex and detailed compositions.

Start a new drawing using the Arch-Imperial template. Right-click on the OSNAP tab at the bottom of the screen. A pop-up menu appears.

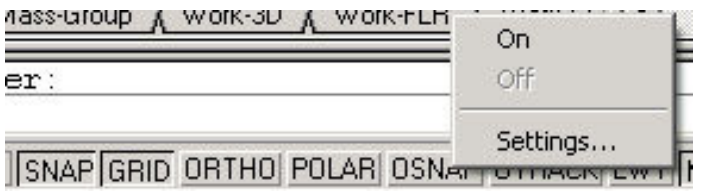

\footnotetext{
"Proceedings of the 2005 American Society for Engineering Education Annual Conference \& Exposition Copyright (c) 2005, American Society for Engineering Education"
} 